\title{
EPILEPSY
}

\section{High-frequency oscillations pinpoint the seizure-onset zone}

Two new studies highlight the utility of high-frequency oscillations (HFOs) for the precise delineation of the seizureonset zone (SOZ) in patients with focal epilepsy. Measurement of HFOs could potentially form part of the presurgical evaluation process in such individuals.

For the first study, François Dubeau and colleagues selected 16 patients with focal epilepsy who had normal MRI scans. Each patient had previously been found to have least one SOZ. Intracranial EEG was used to measure interictal HFOs $(\geq 80 \mathrm{~Hz})$ and spikes during slow-wave sleep. Both spike and HFO activity was elevated in brain regions identified as
SOZs, although HFOs were more accurate than spikes at pinpointing the SOZ. This study is the first to correlate interictal HFOs with the SOZ in patients with focal epilepsy and normal MRI.

In the second study, Pradeep Modur and colleagues measured ictal HFOs $(\geq 70 \mathrm{~Hz})$ in six patients with neocortical epilepsy, as part of a presurgical planning protocol to identify brain tissue for resection. "We defined the SOZ to include those channels with HFOs that showed subsequent evolution (HFOs+) but not the channels that lacked evolution (HFOs-)," explains Modur. "In contrast to the HFOs-, the HFOs+ were higher

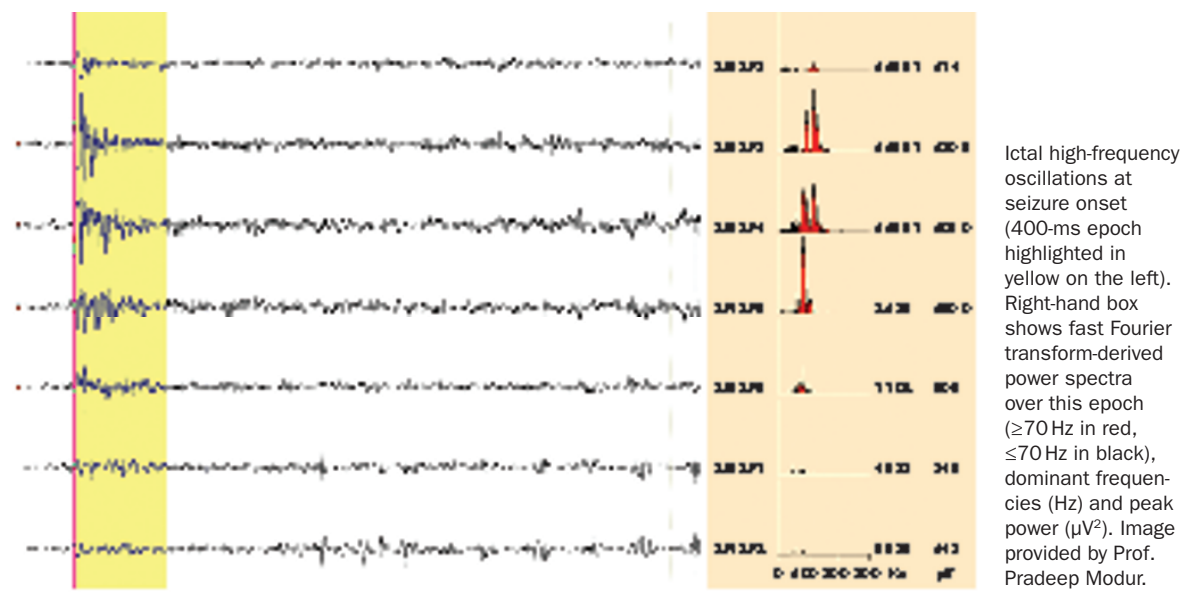

in peak frequency, more robust, and spatially restricted."

The six patients all underwent resection on the basis of ictal HFO data, and five achieved a class I or II outcome (free of disabling seisures or almost seizure-free) over a mean 2 -year follow-up period. The outcome in the remaining patient was less satisfactory, possibly because the $\mathrm{SOZ}$ was incompletely resected owing to overlap with the language area.

"Our study demonstrates the widespread occurrence of ictal HFOs at seizure onset and outlines a method to localize the SOZ based on their restricted pattern of evolution," says Modur. "We have shown that smaller resections, restricted mainly to the HFOs+ channels, can lead to favorable outcome." His team now plans to attempt to replicate the results in a larger group of patients, and to extend their investigations to patients with mesial temporal lobe epilepsy.

\section{Heather Wood}

Original articles Andrade-Valença, L. et al. Interictal high frequency oscillations (HFOs) in patients with focal epilepsy and normal MRI. Clin. Neurophysiol. doi:10.1016/j. clinph.2011.06.004 | Modur, P. N. et al. Ictal highfrequency oscillations in neocortical epilepsy: implications for seizure localization and surgical resection. Epilepsia doi:10.1111/j.1528-1167.2011.03165.x 